\title{
Phonon Anomalies Induced by Superconductivity
}

\author{
Hae-Young Kee ${ }^{1,2}$ and C. M. Varma ${ }^{2}$ \\ ${ }^{1}$ Department of Physics, Rutgers University, Piscataway, NJ 08855-0849 \\ 2 Bell Laboratories, Lucent Technologies, Murray Hill, NJ 07974
}

\begin{abstract}
We calculate the electronic polarizability in the superconducting state near extremum vectors $\vec{Q}_{0}$ of the Fermi surface. A pole appears in the polarizability at frequencies $\omega$ near the superconducting gap $2 \Delta$ which leads to a sharp peak at $\omega$ just below $2 \Delta$ in the lattice vibrations near $\vec{Q}_{0}$. A second order transition to a charge density wave state below the superconducting transition is shown to be unlikely. The results are compared with the recent inelastic neutron scattering measurements in compounds, $A N i_{2} B_{2} C, A=Y$ or $L u$.

74.20.-z,74.25.kc
\end{abstract}

Typeset using REVTEX 
Strong momentum dependence of the electronic polarizability leads to characteristic features in the dispersion relation of phonons in simple metals-the Kohn anomalies. [1] Transition metals and compounds are particularly beset with wiggles and dips in their phonon dispersions. Strong electron-phonon interactions and local field effects are responsible together with nesting features in the band structure for the anomalies and for the occurrence of charge density wave transitions [2].

It is well known that the leading order polarizability $\Pi_{0}^{n}(\vec{Q}, \omega)$, Fig. $1(\mathrm{a})$, of an electron gas in d-dimensions for $\vec{Q}=2 \vec{k}_{F}$ for a spherical Fermi-surface or ellipsoidal Fermi-surface across the major and minor axes of the Fermi-surface in the normal state is [3]

$$
\operatorname{Re} \Pi_{0}^{n}\left(2 \vec{k}_{F}, \omega\right) \simeq N(0)\left[1+(N(0) \omega)^{d-1} \ln \left(\frac{E_{F}}{\omega}\right)\right]
$$

We show the existence of singularities in the polarizability at $\omega \simeq 2 \Delta$ in s-wave superconductors near the extremum vectors of the Fermi-surface which are stronger than those of the normal state at $\omega \rightarrow 0$. By an extremum vector we mean $\vec{Q}_{0}$ such that $\epsilon_{\vec{Q}_{0} / 2}$ and $\epsilon_{-\vec{Q}_{0} / 2}$ are both on the Fermi-surface and for small deviations $|\vec{k}|<<\left|\vec{Q}_{0}\right|$

$$
\begin{aligned}
\epsilon_{\vec{k}-\frac{\vec{Q}_{0}}{2}} & =-v_{F\|\|} \vec{k}_{\|}+\sum_{i} \frac{\vec{k}_{i \perp}^{2}}{2 m_{i \perp}} \\
\epsilon_{\vec{k}+\frac{\vec{Q}_{0}}{2}} & =v_{F \|} \vec{k}_{\|}+\sum_{i} \frac{\vec{k}_{i \perp}^{2}}{2 m_{i \perp}}
\end{aligned}
$$

Here $\vec{k}_{\|}$is the component of $\vec{k}$ parallel to $\vec{Q}_{0}$ and $\vec{k}_{i \perp}$ are the components perpendicular to $\vec{Q}_{0}$ where $i$ runs over $d-1$ values. For example, $\vec{Q}_{0}$ are the major and minor axes for an ellipsoidal Fermi-surface. $\vec{Q}_{0}$ is also a nesting vector when $m_{i \perp}$ for one or more $i$ is infinity.

These singularities strongly affect the phonon spectrum of superconductors under certain conditions. We explain the remarkable difference between the phonon spectrum of $Y \mathrm{Ni}_{2} \mathrm{~B}_{2} \mathrm{C}$ in the superconducting state and that of the normal state recently observed by Kawano et al [4] and in $L u N i_{2} B_{2} C$ by Stassis et al [5]. In the normal phase of $(Y, L u) N i_{2} B_{2} C$ the transverse acoustic and optic dispersion curve in the $\Gamma Z$ direction has a strong temperature dependent dip around $\vec{Q}=(0.5,0,8)$. In the $Y$ compound, below the superconducting transition temperature, the spectral weight splits into two parts, a very sharp resolution 
limited line at an energy $\simeq 2 \Delta$ and a broad part around the phonon peak of the normal state with the total spectral weight conserved. Weight is transferred from the sharp peak to the broad peak at low temperatures by a magnetic field.

As a model, we consider a simple ellipsoidal Fermi-surface, $\epsilon_{\vec{k}}=\sum_{i}^{d} \frac{\vec{k}_{i}^{2}}{2 m_{i}}$, and calculate $\Pi(\vec{Q}, \omega)$ in the superconducting state for $\vec{Q}$ near the extremum vectors $\vec{Q}_{0}$.

We write the model Hamiltonian which describes a system of electron interacting via a potential $\mathrm{V}$,

$$
H_{e l}=\sum_{\vec{k}} \epsilon_{\vec{k}} \Psi_{\vec{k}}^{\dagger} \tau_{3} \Psi_{\vec{k}}+\frac{1}{2} \sum_{\vec{k}, \vec{k}^{\prime}, \vec{Q}} V\left(\vec{k}, \vec{k}^{\prime}, \vec{Q}\right)\left(\Psi_{\vec{k}+\vec{Q}^{\prime}}^{\dagger} \tau_{3} \Psi_{\vec{k}}\right)\left(\Psi_{\vec{k}^{\prime}-\vec{Q}^{\prime}}^{\dagger} \tau_{3} \Psi_{\vec{k}^{\prime}}\right),
$$

in Nambu notation [6], where the electron and annihilation operators are written as twocomponent vectors,

$$
\Psi_{\vec{k}}=\left(\begin{array}{c}
c_{\vec{k} \uparrow} \\
c_{-\vec{k} \downarrow}^{\dagger}
\end{array}\right), \Psi_{\vec{k}}^{\dagger}=\left(c_{\vec{k} \uparrow}^{\dagger}, c_{-\vec{k} \downarrow}\right)
$$

and the $\tau^{\prime}$ 's are Pauli matrices. The potential $V\left(\vec{k}, \vec{k}^{\prime}, \vec{Q}\right)$ includes the electron-electron Coulomb repulsion as well as the attractive interaction mediated by the phonons. As usual [6] we rewrite Eq. (3) as

$$
H_{e l}=H_{0}+H_{1}
$$

where $H_{0}$ is the BCS reduced Hamiltonian,

$$
H_{0}=\sum_{\vec{k}} \Psi_{\vec{k}}^{\dagger}\left(\epsilon_{\vec{k}} \tau_{3}+\Delta \tau_{1}\right) \Psi_{\vec{k}}
$$

and $H_{1}$ now includes the second term of Eq. (3) minus the electron-electron effective interaction absorbed in Eq. (6).

The lowest order polarizability, Fig. 1 (a), at $T=0$ is

$$
\Pi_{0}(\vec{Q}, \omega)=-i \int \frac{d^{3} k d \omega^{\prime}}{(2 \pi)^{4}} \operatorname{Tr}\left[\tau_{3} G\left(\vec{k}+\vec{Q}, \omega+\omega^{\prime}\right) \tau_{3} G\left(\vec{k}, \omega^{\prime}\right)\right]
$$

where $G$ is the single particle Green's function for the BCS reduced Hamiltonian. 


$$
G(\vec{k}, \omega)=\frac{\omega I+\epsilon_{\vec{k}} \tau_{3}+\Delta \tau_{1}}{\omega^{2}-E_{\vec{k}}^{2}+i \delta},
$$

where $E_{\vec{k}}^{2}=\left(\epsilon_{\vec{k}}^{2}+\Delta^{2}\right)$.

To evaluate Eq. (7), it is helpful, after performing the frequency integral, to divide the momentum space into parts in which $E_{\vec{k}}$ and $E_{\vec{k}+\vec{Q}}$ are close to $\Delta$, say between $\Delta$ and $s \Delta, s \approx O(2)$, and those in which they are larger than $s \Delta$. In the latter $E_{\vec{k}, \vec{k}+\vec{Q}}$ may be replaced by $\epsilon_{\vec{k}, \vec{k}+\vec{Q}}$, so that the integrand is the same as in the normal state. Any new features expected in the superconducting state can arise only from the singular density of one-particle states for energies near $\Delta$, i.e., from regions of momentum space where both $E_{\vec{k}}$ and $E_{\vec{k}+\vec{Q}}$ are near $\Delta$. The contribution of this region in three dimensions is,

$$
\begin{aligned}
& \operatorname{Re}_{0}^{s c}(\vec{Q}, \omega) \simeq \frac{N(0) \Delta}{E_{F}} \operatorname{sgn}(\delta \omega) \ln \frac{E_{F}}{\sqrt{|\delta \omega| \Delta}} \\
& \operatorname{Im}_{0}^{s c}(\vec{Q}, \omega) \simeq \begin{cases}0 & \delta \omega<0 \\
-\frac{\pi}{2} \frac{N(0) \Delta}{E_{F}} & \delta \omega>0,\end{cases}
\end{aligned}
$$

where $\delta \omega \equiv \omega-2 \Delta\left(1+\xi^{2} q^{2} / 8\right), N(0)=v \frac{\sqrt{m_{i \perp} m_{j \perp} m_{\|} E_{F}}}{2 \pi^{2}}$ where $v$ is the volume of the unit cell, and $q$ defined to be $\left(\vec{Q}-\vec{Q}_{0}\right)_{\|}$satisfies $q \xi<<1$.

In two dimensions, $\operatorname{Re} \prod_{0}^{s c}\left(\vec{Q}_{0}, \omega\right)$ is proportional to $|2 \Delta-\omega|^{-\frac{1}{4}}$ while in one dimension (where the treatment here is quite inadequate) it is proportional to $|2 \Delta-\omega|^{-\frac{1}{2}}$. For nonextremum $\vec{Q}$ spanning the Fermi-surface, $\operatorname{Re} \Pi_{0}^{s c}(\vec{Q}, \omega)$ is proportional to $\ln |2 \Delta-\omega|$ in two dimensions [7] and $|2 \Delta-\omega|^{\frac{1}{2}}$ in three dimensions.

The contribution of the other regions differs from the normal state only through a lower cut off at $s \Delta$ so that $\ln \left(E_{F} / \omega\right)$ in Eq. (1) becomes $\ln \left[E_{F} /(\omega+s \Delta)\right]$. Since we will be concerned with $\omega \approx O(2 \Delta)$, this change is not of consequence, so that

$$
\operatorname{Re}_{0}(\vec{Q}, \omega) \simeq \operatorname{Re} \Pi_{0}^{s c}(\vec{Q}, \omega)+\operatorname{Re}_{0}^{n}(\vec{Q}, \omega)
$$

In the random phase approximation, Fig. 1 (b),

$$
\Pi(\vec{Q}, \omega)=\Pi_{0}(\vec{Q}, \omega) /\left(1+V(\vec{Q}) \Pi_{0}(\vec{Q}, \omega)\right)
$$


The direct electron-electron interaction is more important in $V(\vec{Q})$ in Eq. (11) compared to the phonon-induced interaction because of the cut-off $E_{F}$ in the former compared to Debye frequency in the latter. Vertex correction to Eq. (11) only change the magnitudes calculated here without affecting the singularities. [8] This is unlike the case $\vec{Q} \rightarrow 0$. 9

Since $\Pi_{0}^{s c}(\vec{Q}, \omega)$ is singular for $\vec{Q}=\vec{Q}_{0}$ and $\omega=2 \Delta$ while $\Pi_{0}^{n}(\vec{Q}, \omega)$ is smooth and much larger than $\Pi_{0}^{s c}$ for all $\omega$ except near $2 \Delta$, polarizability is approximately given by

$$
\begin{array}{ll}
\operatorname{Re} \Pi\left(\vec{Q}_{0}, \omega\right) \simeq \operatorname{Re} \Pi^{s c}\left(\vec{Q}_{0}, \omega\right) & \omega \approx 2 \Delta \\
\operatorname{Re} \Pi\left(\vec{Q}_{0}, \omega\right) \simeq \operatorname{Re} \Pi^{n}\left(\vec{Q}_{0}, \omega\right) & \text { elsewhere }
\end{array}
$$

Here $\Pi^{n}\left(\vec{Q}_{0}, \omega\right)$ is the normal state polarizability and

$$
\operatorname{Re} \Pi^{s c}\left(\vec{Q}_{0}, \omega\right) \simeq \frac{-N(0)}{\beta+V \operatorname{Re} \prod_{0}^{s c}\left(\vec{Q}_{0}, \omega\right)}
$$

In Eq. (11), we have used $\operatorname{Re} \Pi_{0}^{n} \simeq-N(0)$ and $\beta \simeq 1-V N(0)$. In three dimensions $\Pi^{s c}(\vec{q}, \omega)$ always has a pole at

$$
\omega=2 \Delta\left(1-e^{-\frac{\beta}{\gamma}}\right)
$$

where $\gamma=\Delta N(0) V / E_{F}<<1$. Note that this pole is very close to $2 \Delta$ and has weight

$$
r \simeq 2 E_{F} e^{-\frac{\beta}{\gamma}} / V \quad<<1
$$

The phonons couple to the electronic charge density,

$$
H_{e l-p h}=\sum_{\vec{k}} g_{Q}\left(b_{\vec{Q}}+b_{-\vec{Q}}^{\dagger}\right) \Psi_{\vec{k}+\vec{Q}}^{\dagger} \tau_{3} \Psi_{\vec{k}}
$$

where $g_{Q}$ is the electron-phonon coupling constant. The phonon spectrum in a metal is described by

$$
D^{-1}(\vec{Q}, \omega)=D_{0}^{-1}(\vec{Q}, \omega)-\Sigma(\vec{Q}, \omega)
$$

where $\Sigma(\vec{Q}, \omega)=g_{Q}^{2} \Pi(\vec{Q}, \omega)$ and $D_{0}(\vec{Q}, \omega)$ contains no renormalization due to electronphonon interactions. Using Eqs. (12) and (13) in Eq. (17), 


$$
\begin{aligned}
D\left(\vec{Q}_{0}, \omega\right)= & {\left[\omega-\omega_{Q_{0}}-\frac{g_{Q_{0}}^{2} r}{\omega-2 \Delta}+i \Gamma_{Q_{0}} \theta(\omega-2 \Delta)\right]^{-1} } \\
& +\left[\omega+\omega_{Q_{0}}+\frac{g_{Q_{0}}^{2} r}{\omega+2 \Delta}-i \Gamma_{Q_{0}}\{1-\theta(\omega+2 \Delta)\}\right]^{-1}
\end{aligned}
$$

where $\omega_{Q_{0}}$ and $\Gamma_{Q_{0}}$ are the phonon frequencies and linewidths of the normal state obtained from the normal contribution $\Pi^{n}\left(\vec{Q}_{0}, \omega\right)$ to $\Pi\left(\vec{Q}_{0}, \omega\right)$. The $\theta$-function in Eq. (18) takes into account that $\operatorname{Im} \Pi\left(\vec{Q}_{0}, \omega\right)=0$ for $\omega<2 \Delta$. Actually, we should include in Eq. (18) also a contribution to $\Gamma_{Q_{0}}$ due to $\operatorname{Im} \Pi^{s c}\left(\vec{Q}_{0}, \omega\right)$ which increases damping of the normal state if $\omega_{Q_{0}}$ is between $2 \Delta$ and $\approx O(4 \Delta)$.

The electron-phonon coupling function $g_{Q_{0}}^{2} \simeq \lambda \bar{\omega} E_{F}$ where $\bar{\omega}$ is the order of the Debye frequency and $\lambda$ is the dimensionless electron-phonon coupling constant, typically of $O(1)$. Then $\left(g_{Q_{0}}^{2} r\right)^{1 / 2}$ is typically much less than $2 \Delta$. In that case and for $\omega_{Q_{0}}>2 \Delta$, Eq. (18) predicts a pole at

$$
\omega \simeq 2 \Delta-\frac{1}{2} g_{Q_{0}}^{2} r /\left(\omega_{Q_{0}}-2 \Delta\right) \equiv \nu
$$

and peak with width $\Gamma_{Q_{0}}$ at

$$
\omega \simeq \omega_{Q_{0}}+\frac{1}{2} g_{Q_{0}}^{2} r /\left(\omega_{Q_{0}}-2 \Delta\right)
$$

The spectral function of the pole near $2 \Delta$ is

$$
S\left(\vec{Q}_{0}, \omega\right) \simeq \frac{g_{Q_{0}}^{2} r}{4\left(\omega_{Q_{0}}-2 \Delta\right)^{2}+g_{Q_{0}}^{2} r} \delta(\omega-\nu),
$$

with the rest in the peak near $\omega_{Q_{0}}$. If $\omega_{Q_{0}}$ is close to $2 \Delta$, the pole is at $2 \Delta-g_{Q_{0}} r^{1 / 2}$ and shares weight equally with the peak. The spectral function, $S\left(\vec{Q}_{0}, \omega\right)=-\frac{1}{\pi} \operatorname{Im} D\left(\vec{Q}_{0}, \omega\right)$ is illustrated in Fig. 2. For $q \neq 0$, the new mode increases in energy by $O\left(\Delta \xi^{2} q^{2} / 4\right)$, and is overdamped for $q>\xi^{-1}$.

In comparing with experimental results 4,5$]$ in $(L u, Y) N i_{2} B_{2} C$, one should bear in mind that the $d=3$ results in normal state are explained in a detailed calculation 11$]$ of $\Pi_{0}(\vec{Q}, \omega)$ based on the actual complicated band structure and phonon renormalizations using electronphonon coupling incorporating local filed effects given by methods of Ref. [2]. We have 
studied only the effects due to superconductivity assuming $\omega_{Q_{0}}$ in Eq. (18) to be the observed normal state value just above $T_{c}$. The band structure of $(Y, L u) N i_{2} B_{2} C$ shows the extremum vectors in the $\left(a^{*}, b^{*}\right)$ plane for $\vec{Q}_{0} \sim(0.5,0,0)$, and weak dependence on momentum in the $c^{*}$ direction. [12] The local field effects quite generally enhance the magnitude of the effects in the superconducting state just as they do in the normal state. But since they are not included in our calculation not too much reliance can be put on the estimate of magnitude of the coefficients in Eqs. (19)-(21). Moreover band structure calculations shows significant nesting near $\vec{Q}_{0}$ of the anomaly. For well nested surfaces, the $d=2$ result quoted below Eq. (9) is relevant and the effects we discuss are correspondingly stronger.

$Y N i_{2} B_{2} C$ is a particularly good candidate for the effects discussed here because the transverse acoustic phonon at $\vec{Q}_{0} \simeq(0.525,0,8)$ softens to an energy $\simeq 7$ meV just above $T_{c}$, not too far above $2 \Delta \simeq 4.3 \mathrm{meV}$. Thus the weight transferred to the peak near $2 \Delta$ is significant and estimated from Eq. (21) to be the experimental value [4] $\approx 0.3$ if $r / 2 \Delta=$ $0.4 N(0)$ and $\gamma \approx O(0.01)$ at $T=0$. Actually, we have no independent way of estimating $r$. This value is reasonable only if $\beta \approx O(0.1)$, which implies a phonon close to an instability in the normal phase, as in these compounds.

A simple extension of the calculation above shows that as the temperature is decreased and $\Delta(T)$ grows, weight is transferred to the sharp peak at the expense of the broad peak with the total weight constant. Similarly a magnetic field depresses the weight and the frequency of the sharp peak. These are as observed [4]. The theory would also predict a temperature dependence to the frequency of the peak $\simeq 2 \Delta(T)$. In the experiment [4], the frequency of the new peak appears not to follow the BCS dependence of $2 \Delta(T)$. This may be so because of strong-coupling effects. Note also that the relative intensity of the peak drops rapidly as its frequency decreases below $2 \Delta(0)$, i.e., near $T_{c}$, making it harder to observe.

We finally comment on other possible explanation of the data. The observed peaks cannot be the large $\vec{Q}$-extensions of the amplitude modes of superconductors [9] near $\vec{Q} \simeq 0$ observed in Raman scattering [13] since such modes are heavily damped for $|\vec{Q}| \geq \xi^{-1}$. 
Another possibility is that the peak is just the narrowing of the linewidth as the phonon frequency goes below $2 \Delta$. This would predict that the number of peaks for a fixed $\vec{Q}_{0}$ as a function of $\omega$ remains the same in the normal state and the superconducting state. In Kawano et al's data [4], an extra new peak for $T<T_{c}$ clearly appears, as in the theory here. In the data of Stassis et al in $L u N i_{2} B_{2} C$ [5], an extra peak is not visible. In this compound the phonon frequency of the soft transverse acoustic mode in the normal state just above $T_{c}$ is lower than that in $Y$-based compound, and in fact close to the sharp peak appearing well below $T_{c}$. Recall that if $\omega_{Q_{0}}$ is close to $2 \Delta(0)$, the two peaks in the superconducting state are split only by $g_{Q_{0}} r^{1 / 2}$ as discussed below Eq. (21). The two peaks may then lie within the experimental resolution of about $1 \mathrm{meV}$. Further experiments are required to elucidate the situation.

An interesting conclusion from our work is that a second order transition to a charge density wave state at a temperature, $T_{C D W}$, below the superconducting transition temperature, $T_{c}$, may not be allowed. A second order transition requires $\omega_{Q_{0}}(T) \rightarrow 0$ as $T \rightarrow T_{C D W}$. But $\omega_{Q_{0}}(T)$ is bounded from below by $2 \Delta(T)$ for $T_{c}>T_{C D W}$, because of the singular contribution to the phonon self-energy as $\omega_{Q_{0}}$ approaches $2 \Delta(T)$. The variation of the phonon frequencies as a function of temperature is schematically illustrated in Fig. 3. A first order transition is, of course, allowed. These matters need further investigation.

In summary, we find the singularities in the polarizability of s-wave superconductor at nesting vectors in three and two dimensions, which manifest themselves as poles in the lattice response function at $\omega$ just below $2 \Delta$ with its weight dependent on the frequency of the normal state phonon at the nesting vector. These results are in general accord with observations. A straightforward extension of these results can be made to d-wave superconductors. If the maximum of the gap function in such superconductors is along the nesting direction, the results for d-wave superconductors are expected to be similar to those obtained here.

We are grateful to Dr. H. Kawano and Prof. C. Stassis for discussions of the experimental results and to Prof. B. Altshuler for pointing out an error in the calculation. We have also 
learned that Dr. S. K. Sinha(private communication) has arrived at conclusions similar to those in the present paper. One of authors (H. Y. K.) thanks E. Abrahams, S.-W. Cheong, Y. B. Kim, and P. B. Littlewood for helpful discussions. This work is supported in part by the Korea Research Foundation and NSF Grant No. DMR-96-32294 (H. Y. K.). 


\section{REFERENCES}

[1] W. Kohn, Phys. Rev. Lett. 2, 393 (1959).

[2] C. M. Varma and W. Weber, Phys. Rev. B 19, 6142 (1979); C. M. Varma and A. L. Simons, Phys. Rev. Lett. 51, 138 (1983).

[3] A. L. Fetter and J. D. Walecka, Quantum Theory of Many Particle Systems (McGraw Hill, New York, 1971).

[4] H. Kawano et. al, Phys. Rev. Lett. 77, 4628 (1996).

[5] C. Stassis st. al, unpublished.

[6] Y, Nambu, Phys. Rev. 117, 648 (1960); J. R. Schrieffer, Theory of Superconductivity (Benjamin/Cummings, Reading, Massachusetts, 1964).

[7] I. I. Mazin and V. M. Yakovenko, Phys. Rev. Lett. 75, 4134 (1995).

[8] As has been noted before, $\Pi_{0}(\vec{Q}, \omega)$ for $\vec{Q} \simeq 0$ has an inverse square root singularity at $\omega=2 \Delta$ in all dimensions [9, 10]. But this singularity is removed in a gauge-invariant theory using Coulomb interaction for $V(\vec{Q})[9]$. By contrast $V(\vec{Q})$ for large $\vec{Q}$ may be taken to be a constant and only shifts the position of the pole in Eq. (12), so that the vertex correction does not affect the singularity in this case.

[9] P. B. Littlewood and C. M. Varma, Phys. Rev. Lett. 47, 811 (1981); Phys. Rev. B 26, 4883 (1982).

[10] C. A. Balseiro and L. M. Falicov, Phys. Rev. Lett. 45, 662 (1980).

[11] W. Weber, unpublished.

[12] J. Y. Rhee, X. Wang, and B. N. Harmon, Phys. Rev. B 51, 15585 (1995).

[13] R. Sooryakumar and M. V. Klein, Phys. Rev. Lett. 45, 660 (1980). 


\section{Figure Captions}

Fig. 1: (a) Lowest order of polarizability $\Pi_{0}(\vec{Q}, \omega)$, and (b) Polarizability $\Pi(\vec{Q}, \omega)$ in random phase approximation.

Fig. 2: Phonon spectral function $S\left(\vec{Q}_{0}, \omega\right)$ calculated from Eq. (21) for various $\bar{\omega}_{Q_{0}}$ where $\bar{\omega}_{Q_{0}}=\omega_{Q_{0}} / 2 \Delta$, and $r / 2 \Delta=0.4 N(0)$.

Fig.3: Schematic behavior of the phonons as a function of temperature. For $T_{c}$ below the charge density wave transition temperature $T_{C D W}$, there is only a single branch $\omega_{Q_{0}} \rightarrow 0$ as $T \rightarrow T_{C D W}$, as shown by the dashed line. For $T_{c}>T_{C D W}$, the soft phonon branch splits into two for $T<T_{c}$, the lower one $\omega_{1}$ is slightly below $2 \Delta(T)$ and the upper one, $\omega_{2}$ considerably broadened with its lower edge at slightly above $2 \Delta(T)$. 


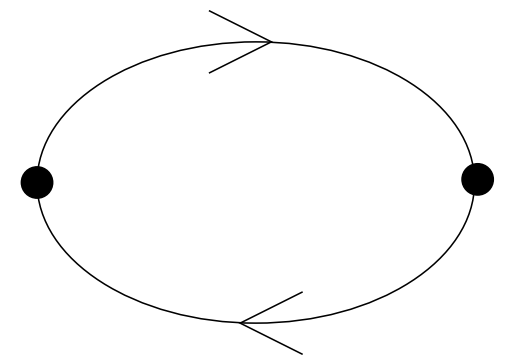

Fig. 1 (a)

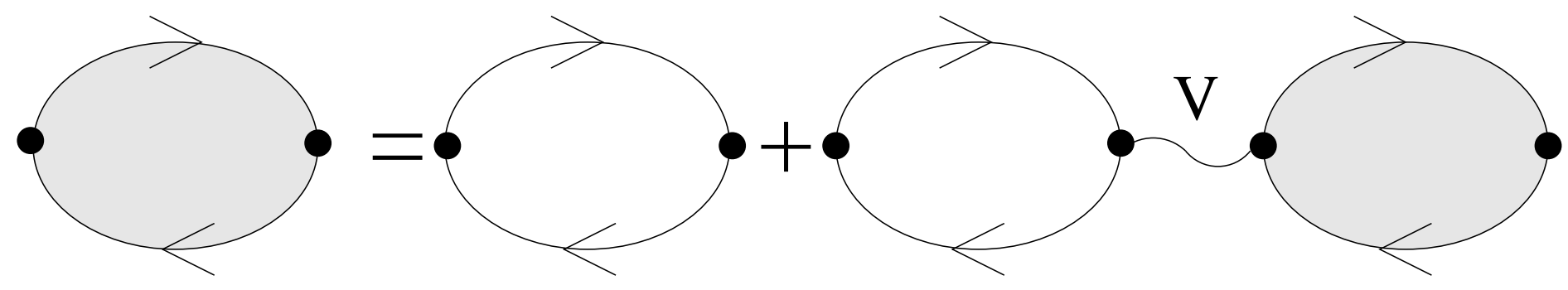

Fig. 1 (b) 


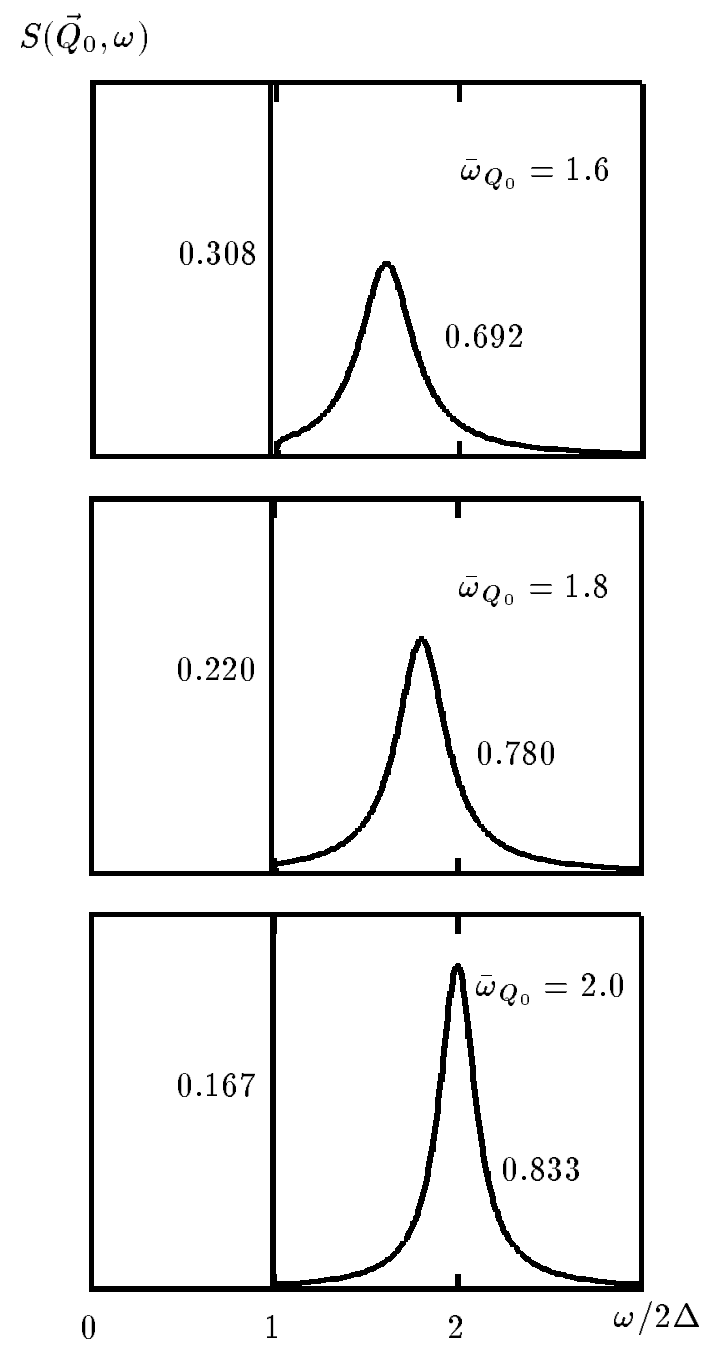

Fig. 2 


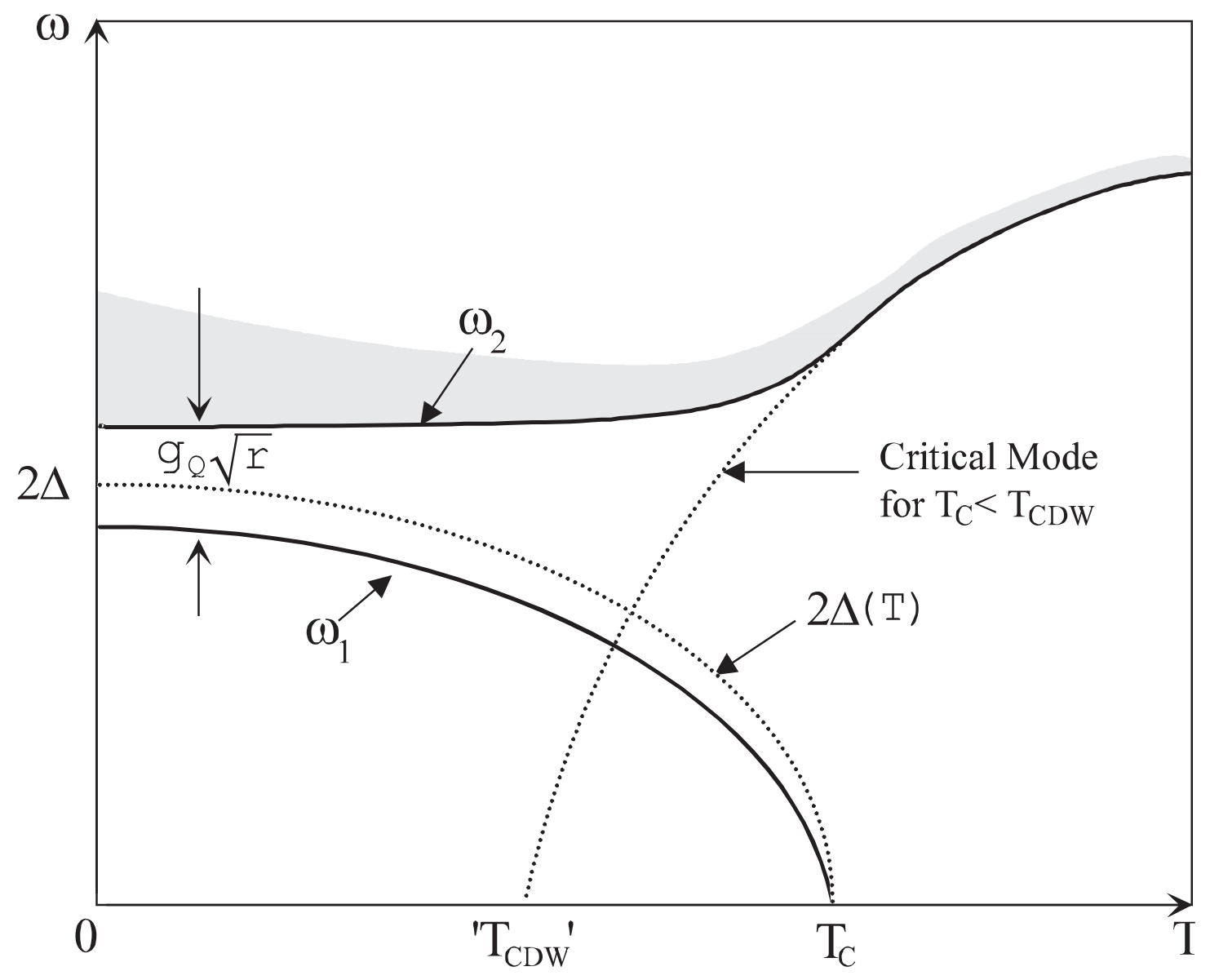

